\title{
Heuristic Algorithms for Solving Bounded Diameter Minimum Spanning Tree Problem and Its Application to Genetic Algorithm Development
}

\author{
Nguyen Duc Nghia and Huynh Thi Thanh Binh \\ Ha Noi University of Technology \\ Viet Nam
}

\section{Introduction}

The bounded diameter minimum spanning tree (BDMST) problem is a combinatorial optimization problem that appears in many applications such as wire-based communication network design when certain aspects of quality of service have to be considered, in ad-hoc wireless network (K. Bala, K. Petropoulos, T.E. Sterm, 1993) and in the areas of data compression and distributed mutual exclusion algorithms (K. Raymond, 1989; A. Bookstein, S. T. Klein, 1996). A more comprehensive discussion of the real-world applications of BDMST was given in Abdalla's seminal dissertation (Abdalla, 2001).

Before the BDMST problem can be formally stated, we need some definitions relating to tree diameter and center. Given a tree $T$, the maximal eccentricity of vertex $v$ is the length (measured in the number of edges) of the longest path from $v$ to other vertices. The diameter of a tree $T$, denoted as diam $(T)$, is the maximal eccentricity over all nodes in $T$ (i.e the length of maximal path between two arbitrary vertices in $T$ ). Suppose that a diameter of a tree is defined by the path $v_{0}, v_{1}, v_{2}, \ldots, v_{[\mathrm{k} / 2]}, v_{[\mathrm{k} / 2]+1}, \ldots, v_{\mathrm{k}}$. If $\mathrm{k}$ is even then $v_{[\mathrm{k} / 2]}$ is called a center of the tree. If $\mathrm{k}$ is odd then $v_{[\mathrm{k} / 2]}$ and $v_{[\mathrm{k} / 2]+1}$ are centers of the tree. In that case, the edge $\left(v_{[\mathrm{k} / 2]}\right.$, $\left.v_{[\mathrm{k} / 2]+1}\right)$ is called a center edge.

Let $G=(V, E)$ be a connected undirected graph with positive edge weights $w(e)$. The BDMST problem can be formulated as follows: among spanning trees of $G$ whose diameters do not exceed a given upper bound $k \geq 2$, find the spanning tree with the minimal cost (sum of the weights on edges of the trees). As in almost all studies of the BDMST problem, and without lost of generality, we will assume that $G$ is a complete graph.

More precisely, the problem can be stated as:

Find a spanning tree $T$ of $G$ that minimizes

$$
W(T)=\sum_{e \in T} w(e)
$$

subject to

$$
\operatorname{diam}(T) \leq k
$$


This problem is known to be $N P$-hard for $4 \leq k<|V|-1$ (M.R.Garey \& D.S.Johnson, 1979). In this chapter, we introduce the heuristic algorithms for solving BDMST: OTTC (Abdall, 2001), $R G H$ (R.Raidl \& B.A.Julstrom, 2003), $R G H_{1}$ (Binh et.at, 2008a), $R G H-I$ (A. Singh \& A.K. Gupta, 2007), CBRC (Binh et al., 2008b). In order to inlustrate the effectiveness of the proposed algorithms, we apply them for initializing the population of our new genetic algorithm with multi-parent recombination operator for solving given problem. Then results of computational experiments are reported to show the efficiency of proposed algorithms.

The chapter is organized as follows. In the next section (section 2), we briefly overview works done in solving BDMST problems. Section 3 deals with new heuristic algorithm for solving BDMST problem. Section 4 describes our new genetic algorithm which uses heuristic algorithms that already presented in previous section to solve BDMST problem. The details of experiments and the comparative computational results are given and discussed in the last section of the chapter.

\section{Previous work on the BDMST problem}

Techniques for solving the BDMST problem may be classified into two categories: exact methods and inexact (heuristic) methods. Exact approaches for solving the BDMST problem are based on mixed linear integer programming (N.R.Achuthan et al., 1994), (L Gouveia et al., 2004). More recently, Gruber and Raidl suggested a branch and cut algorithm based on compact 0-1 integer linear programming (M. Gruber \& G.R. Raidl, 2005). However, being deterministic and exhaustive in nature, these approaches could only be used to solve small problem instances (e.g. complete graphs with less than 100 nodes).

(Abdalla et al., 2000) presented a greedy heuristic algorithm - the One Time Tree Construction (OTTC) for solving the BDMST problem. OTTC is based on Prim's algorithm in (R. Prim, 1957). It starts with a set of vertices, initially containing a randomly chosen vertex. The set is then repeatedly extended by adding a new vertex that is nearest (in cost) to the set, as long as the inclusion of the new node does not violate the constraint on the diameter of the tree. This algorithm is time consuming, and its performance is strongly dependent on the starting vertex.

Raidl and Julstrom proposed in (G.R. Raidl \& B.A. Julstrom, 2003) a modified version of OTTC, called Randomized Greedy Heuristics $(R G H)$. RGH starts from a centre by randomly selecting a vertex and keeping it as the fixed center during the search. It then repeatedly extends the spanning tree from the center by adding a randomly chosen vertex from the remaining vertices, and connecting it to a vertex that is already in the tree via an edge with the smallest weight. The obtained results showed that on Euclidean instances $R G H$ performs better than OTTC, whereas on non-Euclidean instances the situation is reversed.

RGH could be summarized in the following pseudo-code (G.R. Raidl \& B.A. Julstrom, 2003)

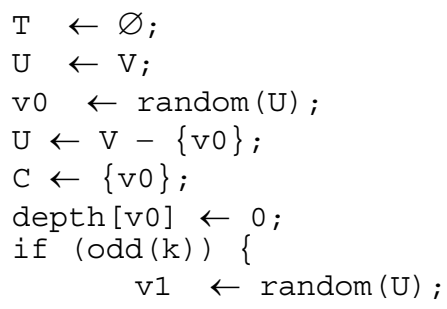




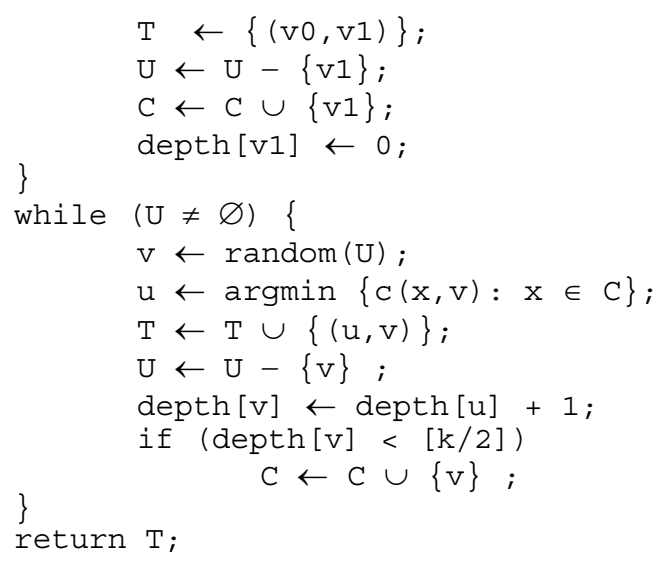

Raidl and Julstrom proposed a genetic algorithm for solving BDMST problems which used edge-set coded (G.R. Raidl \& B.A. Julstrom, 2003) (JR-ESEA) and permutation-coded representations for individuals (B.A. Julstrom \& G.R. Raidl, 2003) (JR-PEA). Permutationcoded evolutionary algorithms were reported to give better results than edge-set coded, but usually are much more time consuming. Another genetic algorithm, based on a random key representation, was derived in (B.A. Julstrom, 2004), sharing many similarities with the permutation-coded evolutionary algorithms. In (M. Gruber \& G.R. Raidl, 2005), Gruber used four neighbourhood types to implement variable neighbourhood local search for solving the BDMST problem. They are: arc exchange neighbourhood, level change neighbourhood, node swap neighbourhood, and center change level neighbourhood. Later, (M. Gruber et al., 2006), re-used variable neighbourhood searches as in (M. Gruber \& G.R. Raidl, 2005), embedding them in Ant Colony Optimization (ACO) and genetic algorithms for solving the $B D M S T$ problem. Both of their proposed algorithms (ACO and GA) exploited the neighbourhood structure to conduct local search, to improve candidate solutions. In (Nghia \& Binh, 2007), Nghia and Binh proposed a new recombination operator which uses multiple parents to do the recombination in their genetic algorithm. Their proposed crossover operator helped to improve the minimum and mean weights of the evolved spanning trees. More recently, in (A. Singh \& A.K. Gupta, 2007), Alok and Gupta derived two improvements for RGH heuristics (given in (G.R. Raidl \& B.A. Julstrom, 2003)) and some new genetic algorithms for solving BDMST problems (notably the GA known as $P E A-I$ ). RGH-I in (A. Singh \& A.K. Gupta, 2007) iteratively improves the solution found with $R G H$ by using level change mutation. It was shown in (A. Singh \& A.K. Gupta, 2007) that RGH-I has better results than all previously-known heuristics for solving the BDMST problem. PEA-I employs a permutation-coded representation for individuals. It uses uniform orderbased crossover and swap mutation as its genetic operators. PEA-I was shown to be the best GA of all those tried on the BDMST problem instances used in (A. Singh \& A.K. Gupta, 2007). In (Binh et al., 2008a), Binh et al., also implement another variant of $R G H$, which is called $R G H_{1} . R G H_{1}$ is similar to $R G H$, except that when a new vertex is added to the expanding spanning tree, it is chosen at random, and connected to a randomly chosen vertex that is already in the spanning tree. 


\section{New greedy heuristic algorithm (center-based recursive clustering)}

Our new greedy heuristics is based on RGH in (G.R. Raidl \& B.A. Julstrom, 2003) and NRGH in (Nghia and Binh, 2007), called CBRC. We extend the concept of center to every level of the partially constructed spanning tree. The algorithm can be seen as recursively clustering the vertices of the graph, in that every in-node of the spanning tree is the center of the subgraph composed of nodes in the subtree rooted at this node. It is inspired from our observation (and other such as in (A. Abdalla et.al, 2000), (G.R. Raidl and B.A. Julstrom, 2003) that good solutions to the BDMST problem usually have "star-like structures" as can be seen (for a Euclidean graph) in Figure 1.

In a star-like structure, the vertices of the graph are grouped in clusters, and the clusters are connected by a link between their centers. Pseudocode for the new heuristic based on this observation, known as Center-Based Recursive Clustering $(C B R C)$, is presented below:

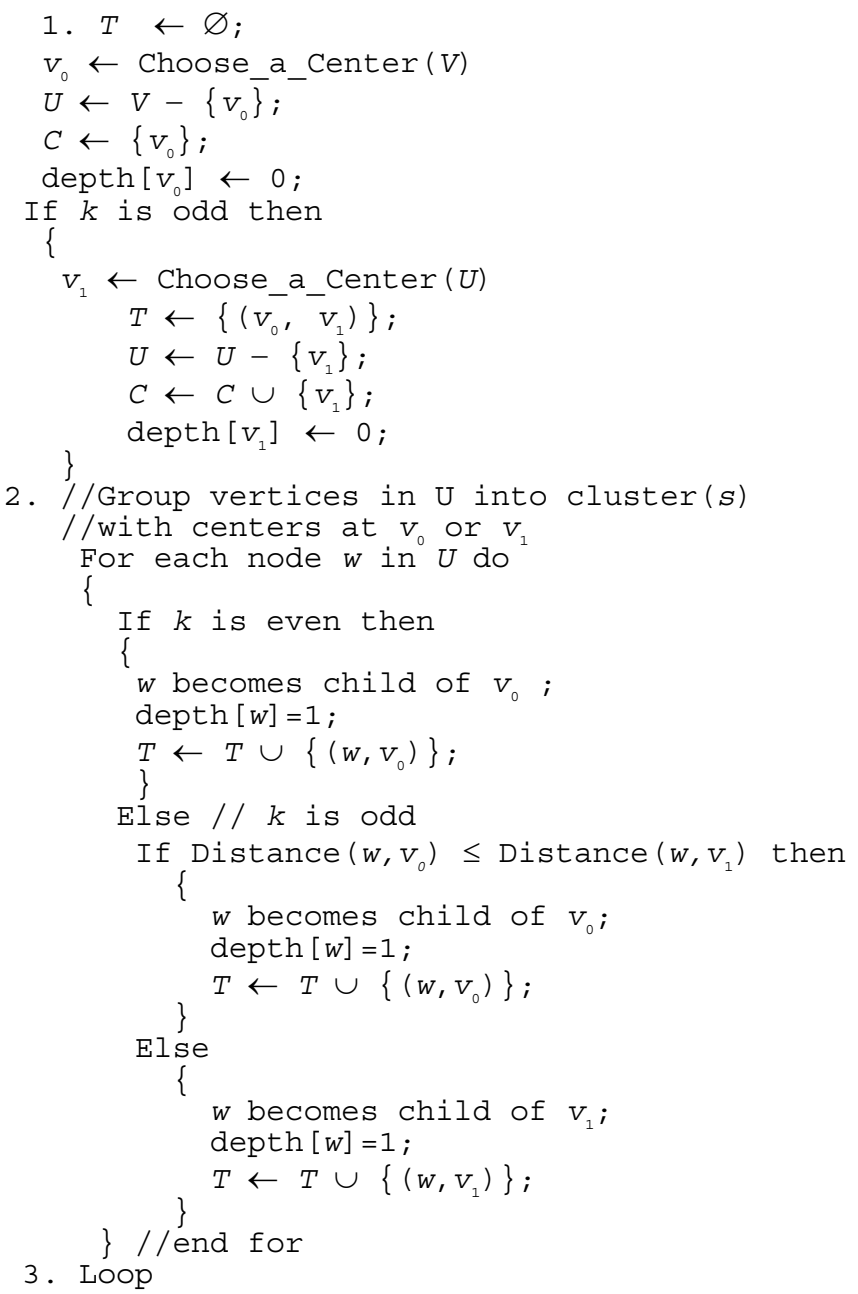




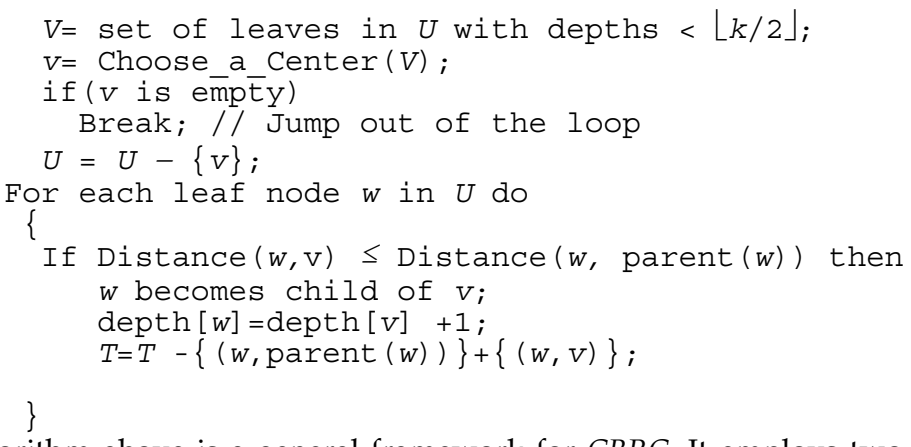

The algorithm above is a general framework for CBRC. It employs two abstract functions, namely, Choose_a_Center and Distance. The implementations of these functions are expected to affect the performance of the heuristics, and the best choice could depend on the problem instance. We propose below some possible implementations of these two functions.

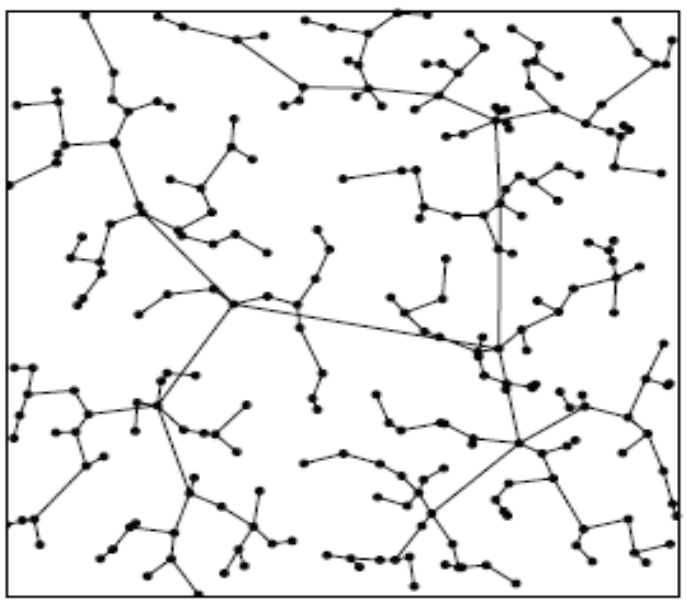

Fig. 1. A "star-like" structure of a typical solution to the BDMST problem.

Implementations of Choose_a_Center function:

- $\quad v$ is a center of $U$ if $\sum w \in U$ Distance $(v, w) \rightarrow$ min. If there is more than one such $v$ then choose from them randomly.

- $\quad$ Rank all vertices in $U$ according to $\sum w \in U$ Distance $(v, w)$, then choose $v$ randomly from the first $h \%$ of the vertices.

- $\quad$ Conduct $h$-tournament selection, $\sum w \in U$ Distance $(v, w)$ as the vertex for $v$.

- $\quad$ Choose $v$ randomly (i.e. it does not depend on Distance at all).

Implementations of the Distance function:

- $\quad \operatorname{Distance}(u, v)=c(u, v)$.

- Distance $(u, v)=$ cost the of shortest path between $u$ and $v$ (used for Non-Eclidean graphs).

It can be seen from the pseudo-code of $C B R C$ that none of the combinations of Distance and Choose_a_Center from the above implementations increase the asymptotic computational complexity of the heuristic to more than $O\left(n^{3}\right)$. It is also possible to apply post- 
improvement, as proposed in (A. Singh and A.K. Gupta, 2007) to CBRC just as for RGH. The resulting heuristic is known as CBRC-I. In the next section, $C B R C$ is tested on some benchmark Euclidean instances of the BDMST problem.

\section{Proposed genetic algorithm}

Genetic algorithm has proven effective on NP-hard problem. Much works research on NPhard problem, particularly in problems relating to tree have been done. Several studies proposed representations for tree (J.Gottlieb et al., 2000), (G.R.Raidl \& B.A.Julstrom, 2003), (B.A.Julstrom \& G.R.Raild, 2003), (B.A.Julstrom, 2004), (Martin Gruber et al., 2006), (Franz Rothlauf, 2006). This section presents the genetic algorithm for solving BDMST problem.

\subsection{Initialization}

Use OTTC, $R G H_{1}, C B R C, R G H$ heuristic algorithms described above for initializing population and edge list for chromosome code.

\subsection{Recombination operator}

Using $k$-recombination operator as in (Nghia and Binh, 2007).

\subsection{Mutation operator}

Using four mutations operators: edge delete mutation, center move mutation, greedy edge replace mutation, subtree optimize mutation as in (G.R.Raidl \& B.A.Julstrom, 2003).

\section{Computational results}

\subsection{Problem instances}

The problem instances used in our experiments are the BDMST benchmark problem instances used in (G.R. Raidl \& B.A. Julstrom, 2003), (A. Singh \& A.K. Gupta, 2007), (Nghia $\&$ Binh, 2007), (Binh et al., 2008a) . They are Euclidean instances. All can be downloaded from http://www.sc.snu.ac.kr/ xuan/BDMST.zip. Euclidean instances are complete random graphs in the unit square. We chose the first five instances of each problem size on Euclide instances (number of vertices) $n=100,250,500$, and 1000, the bounds for diameters being 10, 15, 20, 25 correspondingly (making up 20 problem instances in total).

\subsection{Experiment setup}

We created two sets of experiments. In the first set of experiment, we compare the performance of the heuristic algorithms: OTTC, $R G H, R G H_{1}, C B R C$. The detail of the comparison between other heuristic algorithm for solving BDMST problem such as CBTC, RGH-I, CBRC-I can be refered to (Binh et al., 2008b), (A. Singh and A.K. Gupta, 2007).

There are several heuristic algorithms for solving BDMST problem as mentioned above but no research has concerned with their effectiveness in application to develop hybrid genetic algorithm. Therefore, in second set of experiment, we will try to fix this problem.

In the second set of experiment, we tested six genetic algorithm algorithms for solving $B D M S T$ problem. All of the genetic algorithms use recombination and mutation operator mentioned in section 4 but initialized by different heuristic algorithm. $G A_{1}, G A_{2}, G A_{3}$ uses 
CBRC, OTTC, $R G H_{1}$ algorithm correspondent for initializing the population. $G A_{4}$ uses $C B R C, O T T C, R G H_{1}, R G H$ for initialization the population with the same rate for each heuristic. $G A_{5}$ uses $R G H_{1}, C B R C$ for initializing, the rate of them in the population are 30 and 70. $G A_{6}$ uses $R G H_{1}, O T T C, C B R C$ for initializing, the rate of them in the population are 35,35 and 30 .

\begin{tabular}{|c|c|c|c|c|c|c|}
\hline & $G A_{1}$ & $G A_{2}$ & $G A_{3}$ & $G A_{4}$ & $G A_{5}$ & $G A_{6}$ \\
\hline$C B R C$ & $100 \%$ & $0 \%$ & $0 \%$ & $25 \%$ & $70 \%$ & $30 \%$ \\
\hline$R G H$ & $0 \%$ & $0 \%$ & $0 \%$ & $25 \%$ & $0 \%$ & $0 \%$ \\
\hline OTTC & $0 \%$ & $100 \%$ & $0 \%$ & $25 \%$ & $0 \%$ & $35 \%$ \\
\hline$R G H_{1}$ & $0 \%$ & $0 \%$ & $100 \%$ & $25 \%$ & $30 \%$ & $35 \%$ \\
\hline
\end{tabular}

Fig. 2. The rate of the heuristic algorithms use for initialization of the population in each experiment genetic algorithm

\subsection{System setting}

In the first experiment, the system was run 300 times for each instances. In the second experiment, the population size for $G A_{1}, G A_{2}, G A_{3}, G A_{4}, G A_{5}, G A_{6}$ was 100 . The number of generations was 500. All GAs populations used tournament selection of size 3 and crossover rate of 0.5 . The mutation rates for center level change, center move, greedy edge mutation, and subtree optimize mutation were $0.7,0.2,0.8$, and 0.5 respectively.

Each system was allocated 20 runs for each problem instance. All the programs were run on a machine with Pentium 4 Centrino $3.06 \mathrm{GHz}$ CPU using 512MB RAM.

\subsection{Results of computational experiments}

The experiment shows that:

- Figure 3, 4, 5, 6, 7, 8, 9, 10, 11 show that the proposed heuristic algorithm, called $C B R C$ have the best result than $R G H, O T T C, R G H_{1}$. It means that the solution found by $C B R C$ algorithm is the best solution in comparison with the other known heuristic algorithm for solving BDMST problem on all the instances with $n=100,250,500$ and 1000 ( $n$ is the number of vertices).

- Figure 15 shows that the best solution found by $G A_{1}$ have better result about $22 \%$ than the $C B R C$ which is used for initialization the population in $G A_{1}$ on all 20 problem instances.

- $\quad$ Figure 16 shows that sum up of the best solution found by $G A_{2}$ have better result about approximately four times than the OTTC which is used for initialization the population in $G A_{2}$ on all 20 problem instances.

- $\quad$ Figure 17 shows that sum up of the best solution found by $G A_{3}$ have better result about over 10 times than the $R G H_{1}$ which is used for initialization the population in $G A_{3}$ on all 20 problem instances.

- Figure 11 shows that sum up of the best solution found by CBRC have better result about 6.5 times than the OTTC and 17 times than $R G H_{1}$ while the the figure 18 shows that sum up of the best solution found by $G A_{1}$ have better result about $0.8 \%$ times than the $G A_{2}$ and approximately $2 \%$ than $G A_{3}$. 
a)

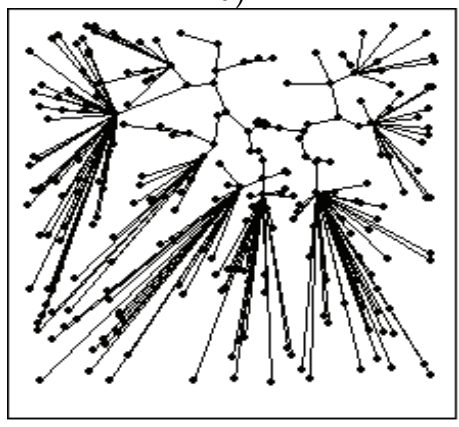

b)

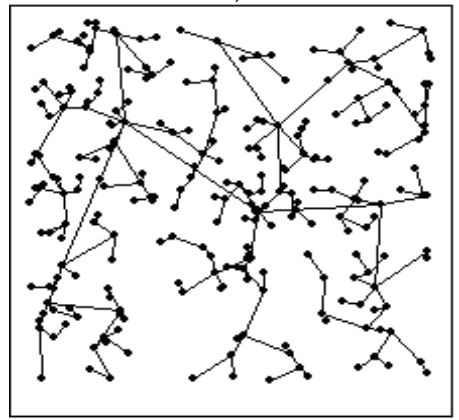

c)

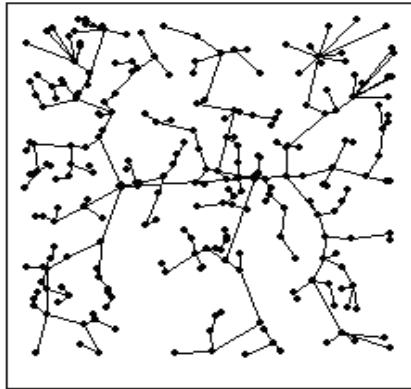

Fig. 3. The best solution found by the heuristics: OTTC, RGH and CBRC on the problem instance with $\mathrm{n}=250$ and $\mathrm{k}=15$, test 1 :

(a) OTTC, weight=42.09; (b) $R G H$, weight=15.14; (c) $C B R C$, weight $=13.32$.

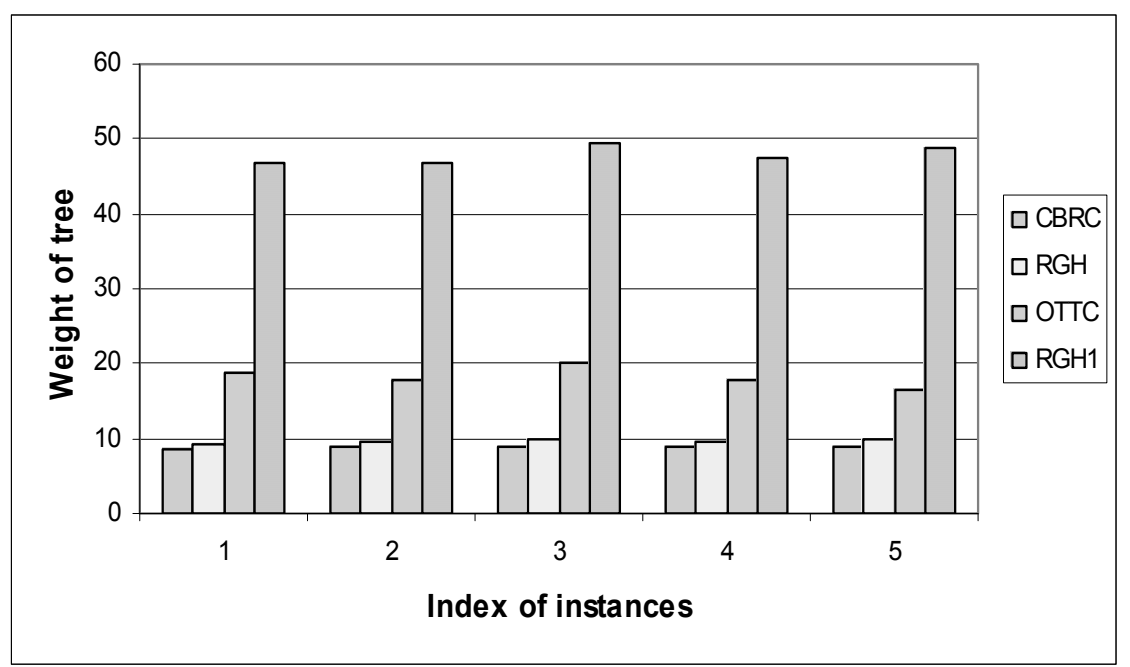

Fig. 4. The best solution found by the four heuristics: $C B R C, R G H, O T T C, R G H_{1}$ on the problem instance with $n=100$ and $k=10$. 


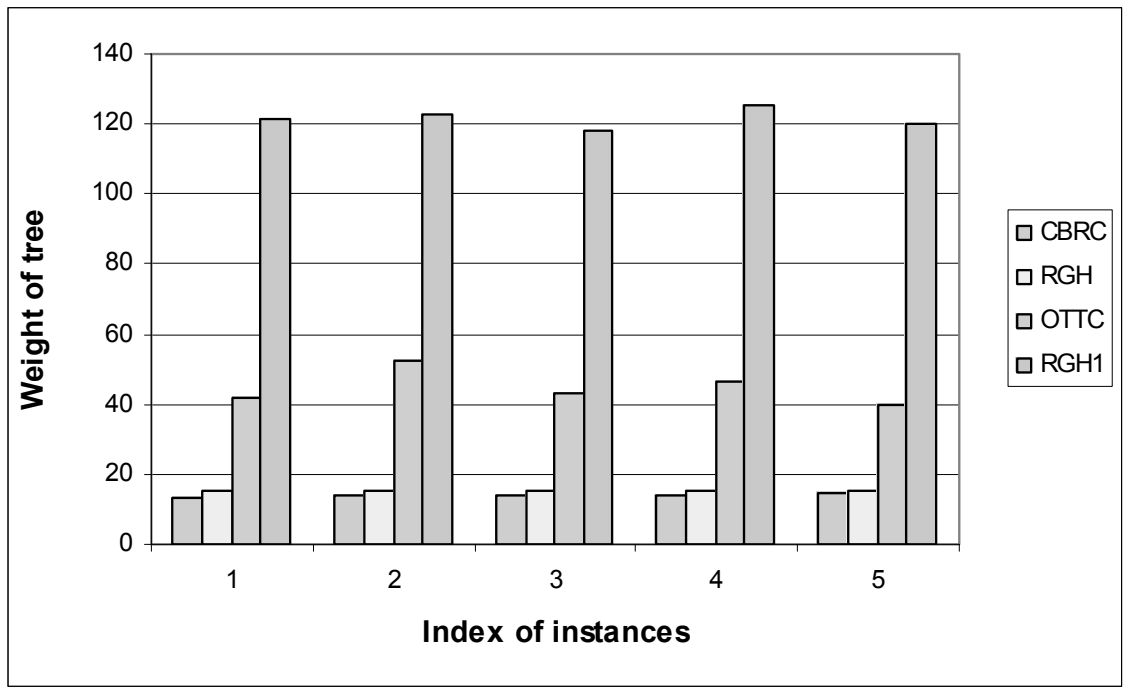

Fig. 5 . The best solution found by the four heuristics: $C B R C, R G H, O T T C, R G H_{1}$ on the problem instance with $n=250$ and $k=15$

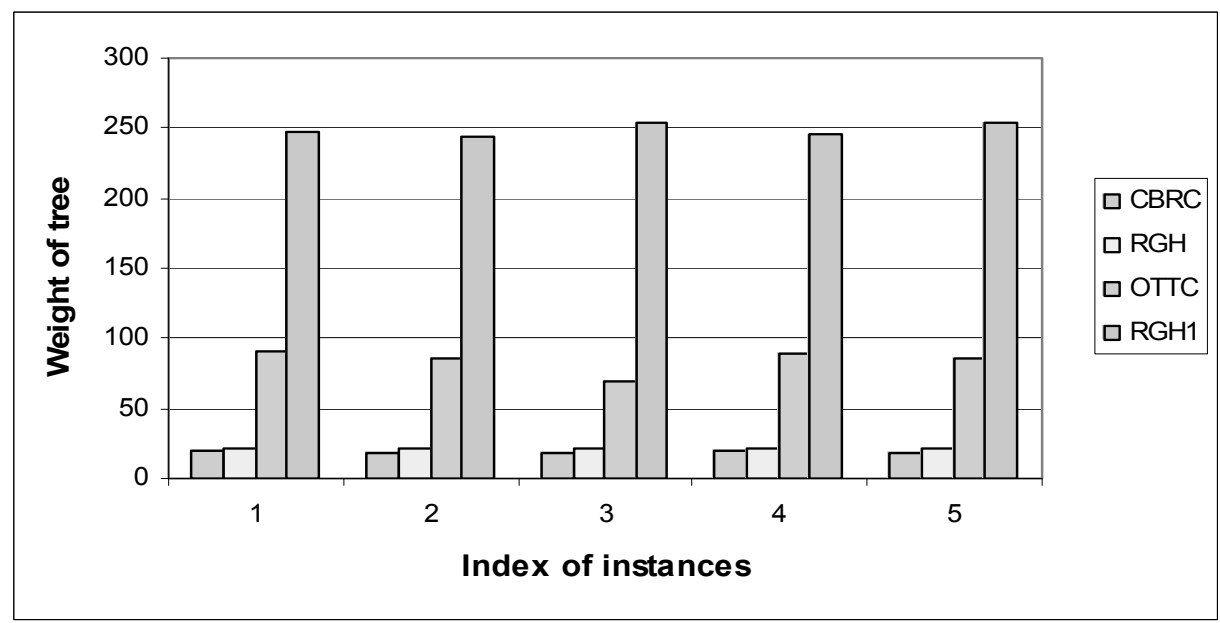

Fig. 6 . The best solution found by the four heuristics: $C B R C, R G H, O T T C, R G H_{1}$ on the problem instance with $n=500$ and $k=20$.

- Figure 18 shows that among $G A_{1}, G A_{2}, G A_{3}, G A_{4}, G A_{5}, G A_{6}$, sum up of the best solution found by $G A_{6}$ have bettest result than the other, otherwise $G A_{3}$ have worest result.

- Figure 19 shows that $G A_{1}$ have smallest sum of standard deviation otherwise $G A_{3}$ have largest sum of standard deviation.

- Figure 20 shows that among $G A_{1}, G A_{2}, G A_{3}, G A_{4}, G A_{5}, G A_{6}$, the number of instances found best result by $G A_{5}$ and $G A_{6}$ are biggest otherwise the number of instances found best result by $G A_{2}$ and $G A_{3}$ are smallest. 


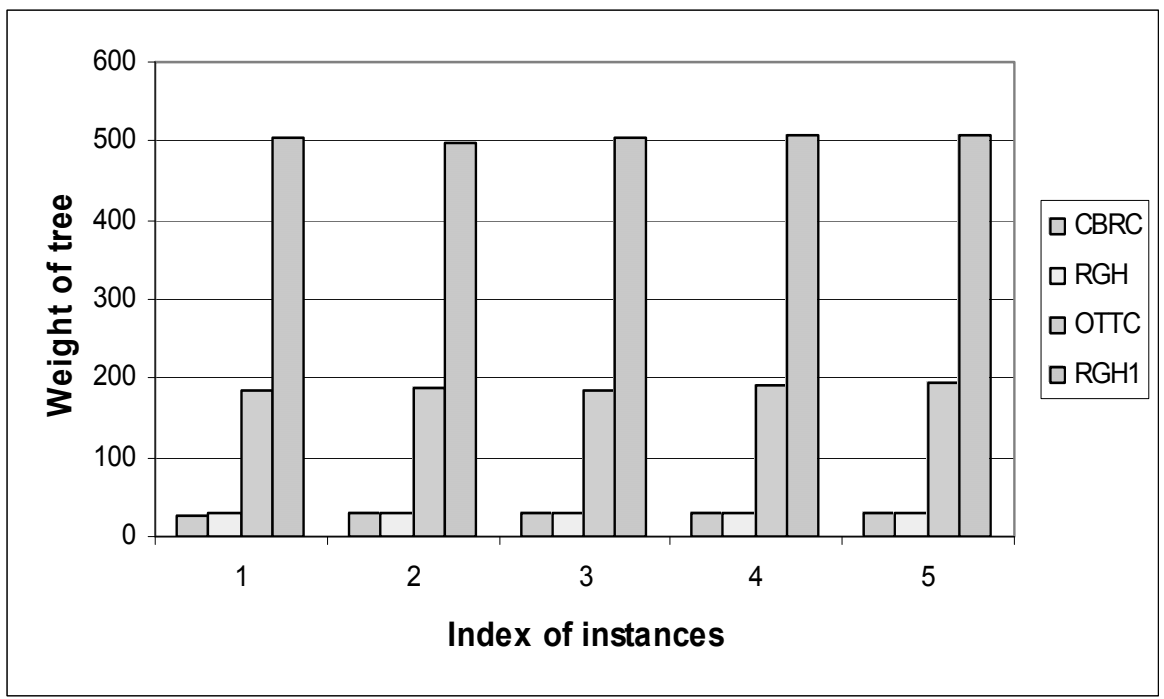

Fig. 7. The best solution found by the four heuristics: $C B R C, R G H, O T T C, R G H_{1}$ on the problem instance with $n=1000$ and $k=25$.

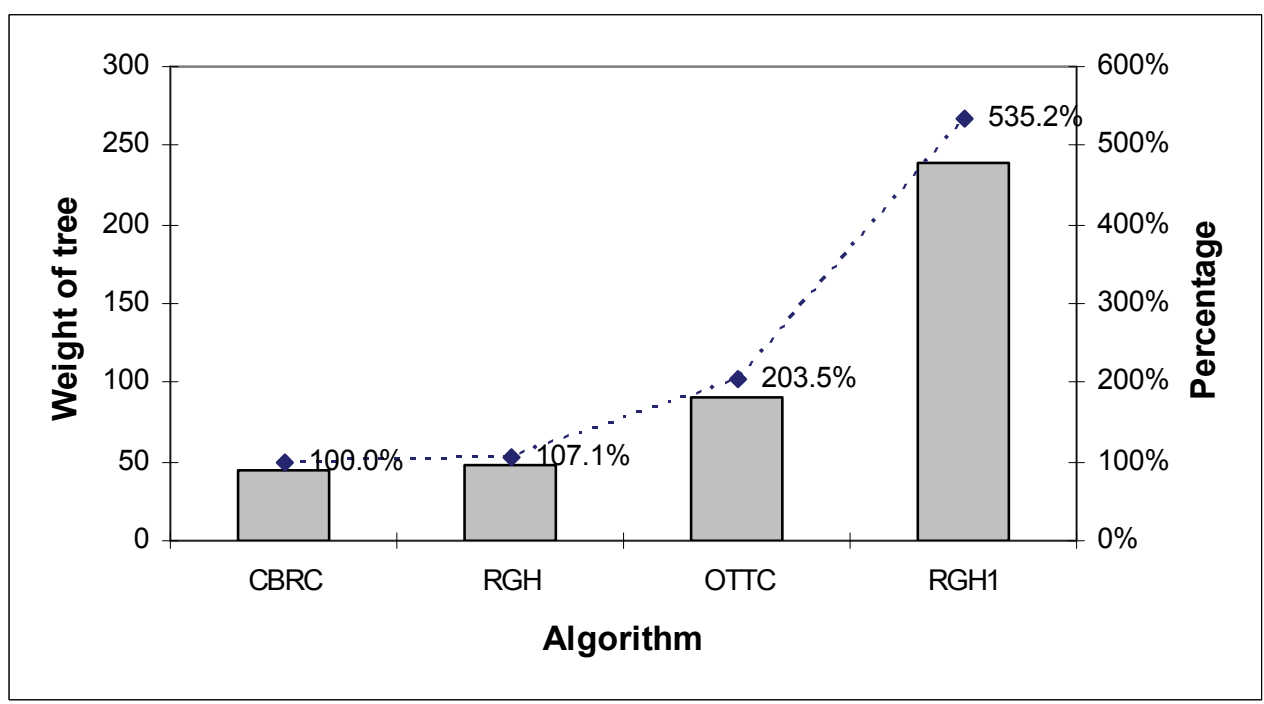

Fig. 8. Comparision of the best solution found by the four heuristics: $C B R C, R G H, O T T C$, $R G H_{1}$ on all the problem instance with $n=100$ (5 instances), $k=10$ 


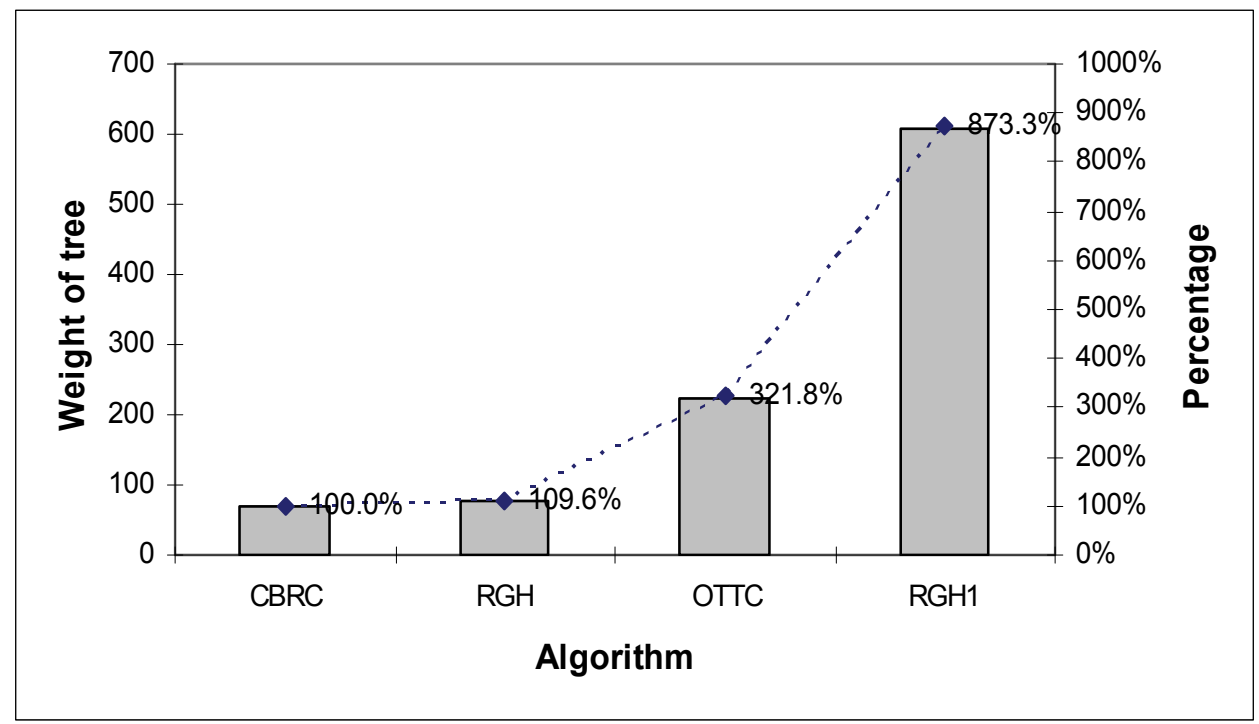

Fig. 9. Comparision between the best solution found by the four heuristics: $C B R C, R G H$, OTTC, $R G H_{1}$ on all the problem instance with $n=250$ ( 5 instances), $k=15$

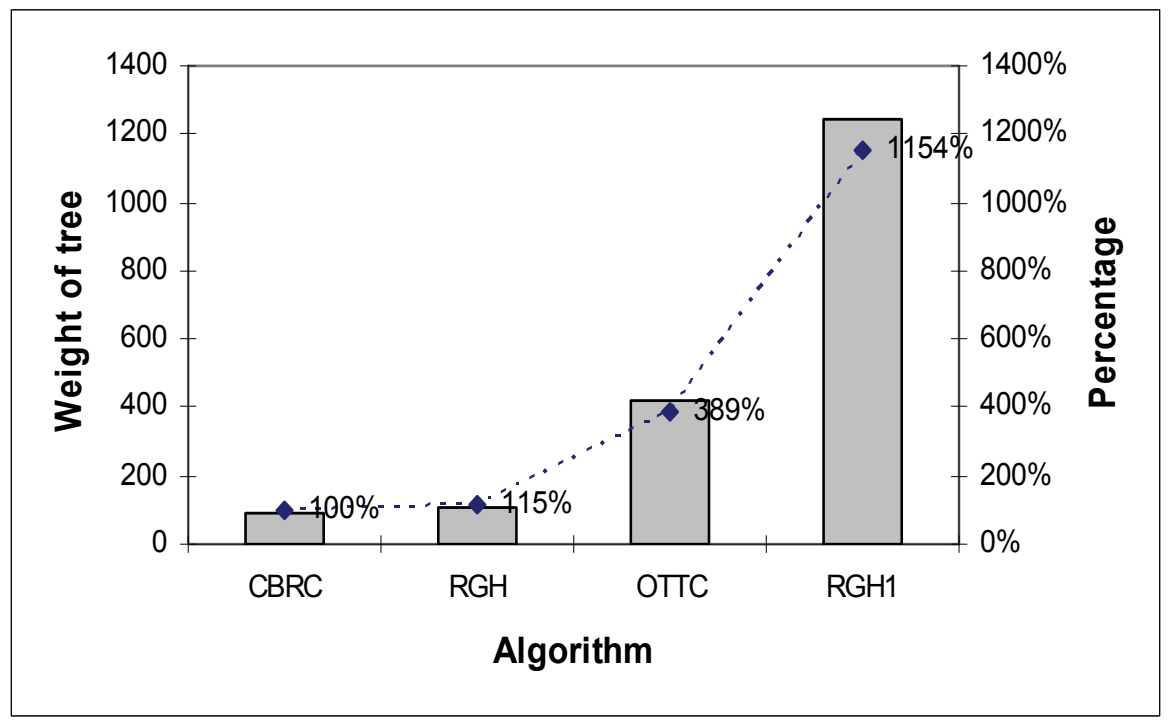

Fig. 10. Comparision between the best solution found by the four heuristics: $C B R C, R G H$, OTTC, $R G H_{1}$ on all the problem instance with $n=500$ ( 5 instances) , $k=20$ 


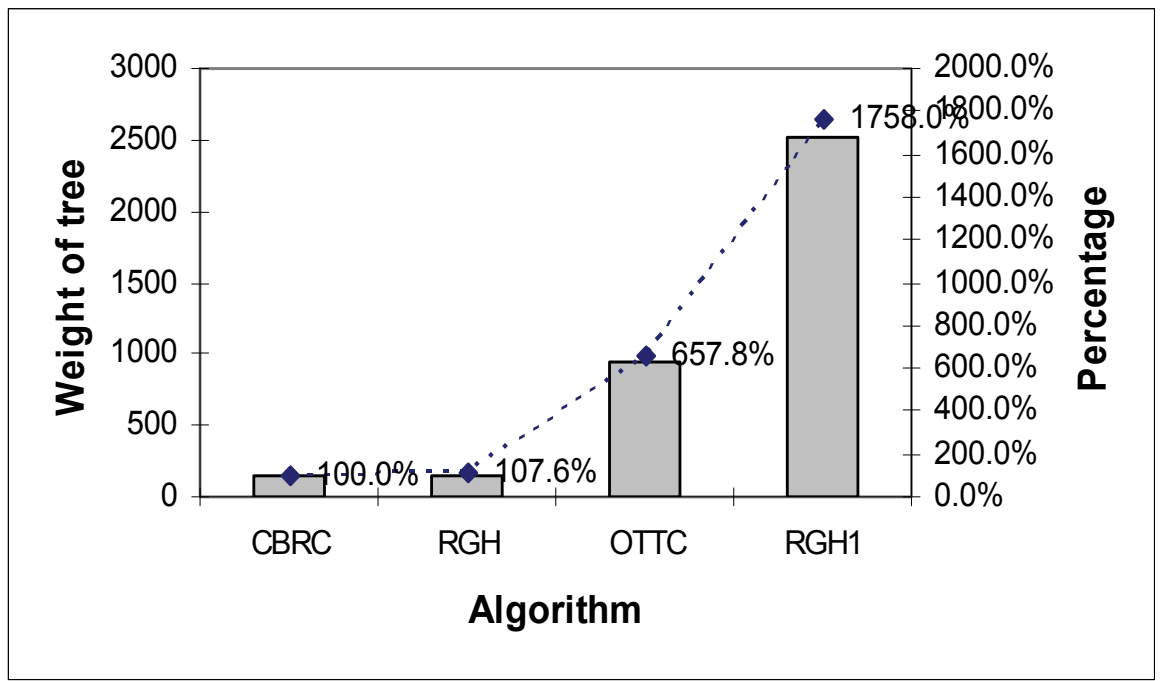

Fig. 11. Comparision between the best solution found by the four heuristics: $C B R C, R G H$, OTTC, $\mathrm{RGH}_{1}$ on all the problem instance with $n=1000$ ( 5 instances) , $k=25$

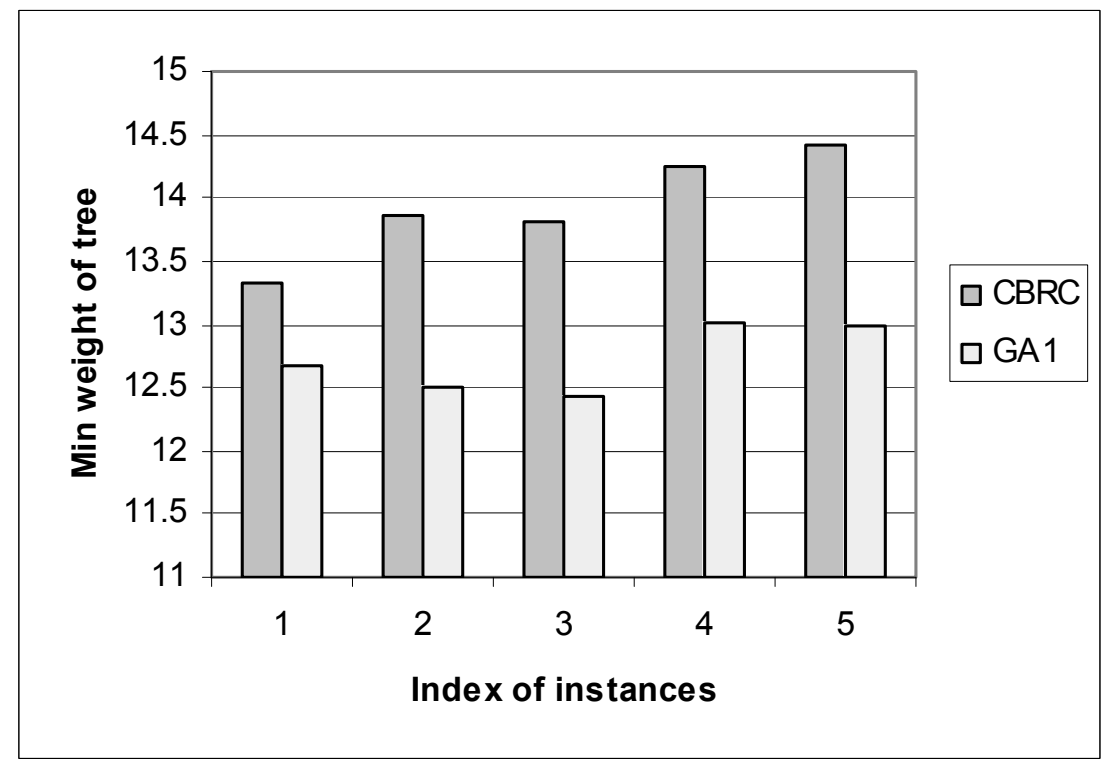

Fig. 12. The best solution found by the $C B R C$ and $G A_{1}$ on all the problem instance with $n=$ $250, k=15$ 


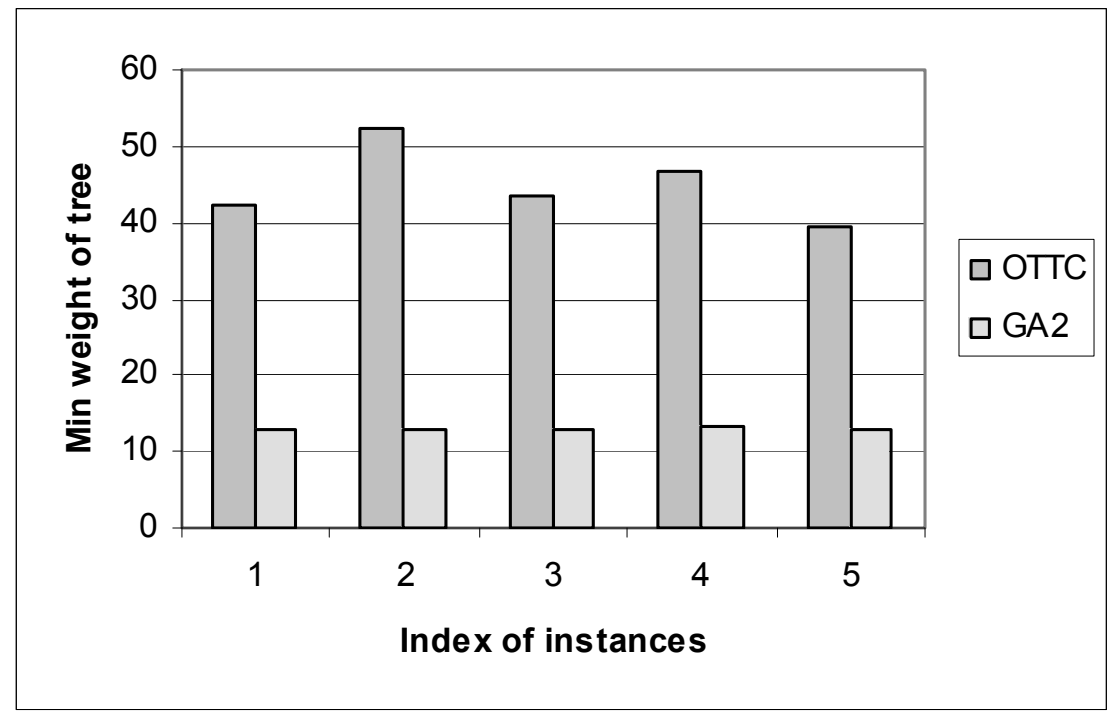

Fig. 13. The best solution found by the OTTC and $G A_{2}$ on all the problem instance with $n=$ $250, k=15$

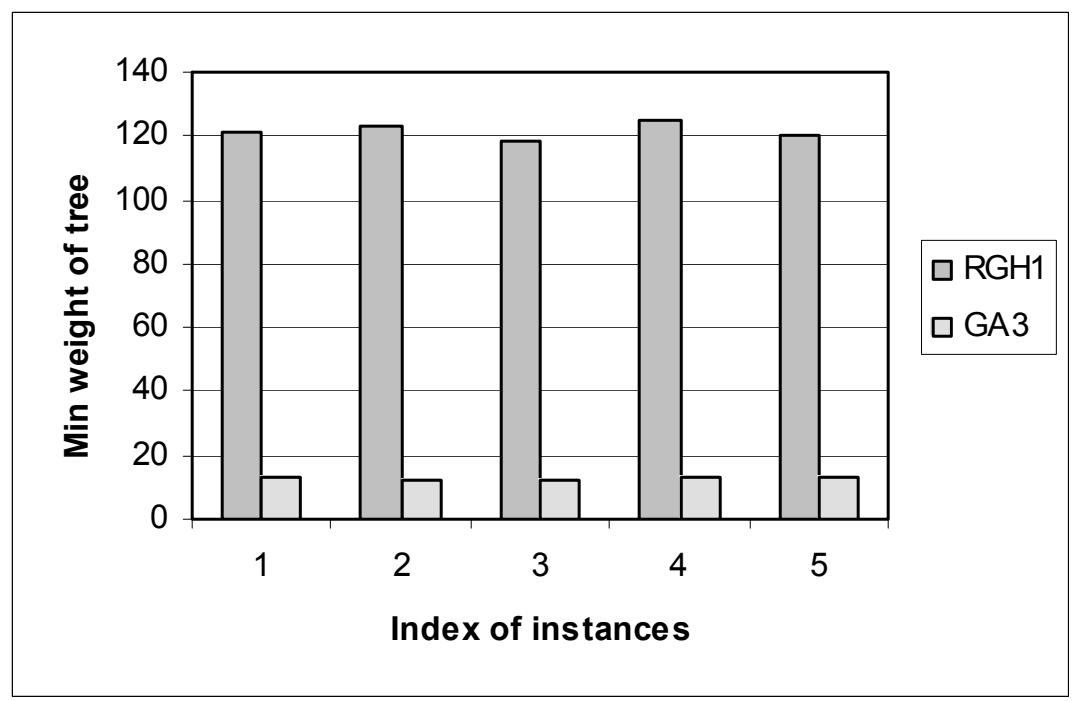

Fig. 14. The best solution found by the $R G H_{1}$ and $G A_{3}$ on all the problem instance with $n=$ $250, k=15$ 


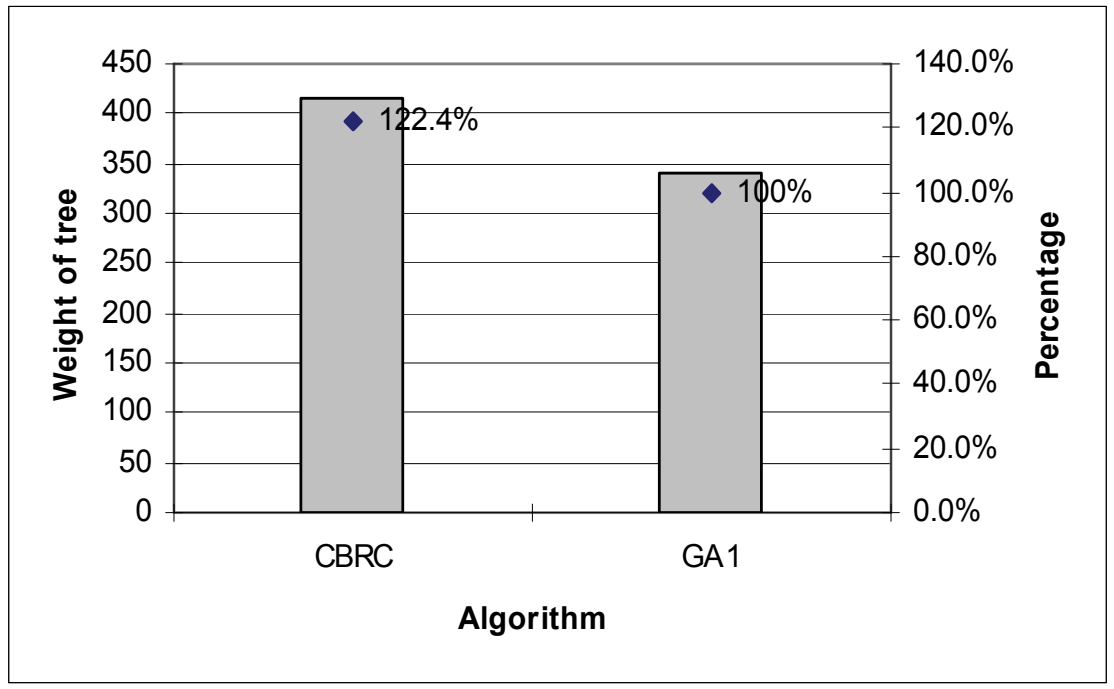

Fig. 15. Sum up the best solution found by the $C B R C$ and $G A_{1}$ on all the problem instances (20 instances)

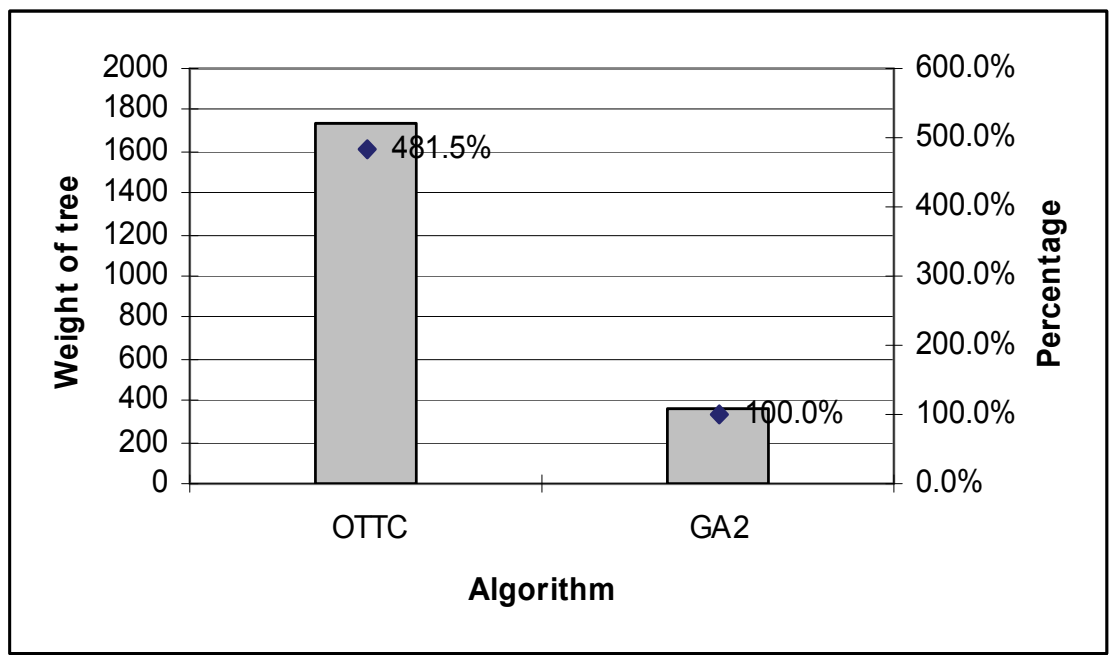

Fig. 16. Sum up the best solution found by the OTTC and $G A_{2}$ on all the problem instances (20 instances) 


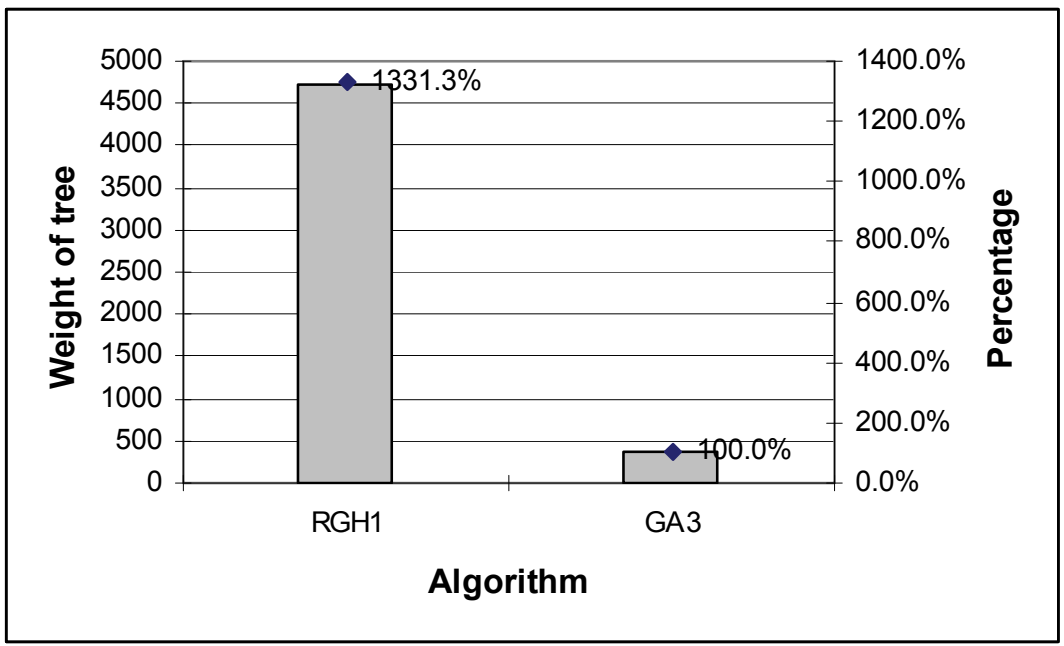

Fig. 17. Comparision between the best solution found by the $R G H_{1}$ and $G A_{3}$ on all the problem instances (20 instances)

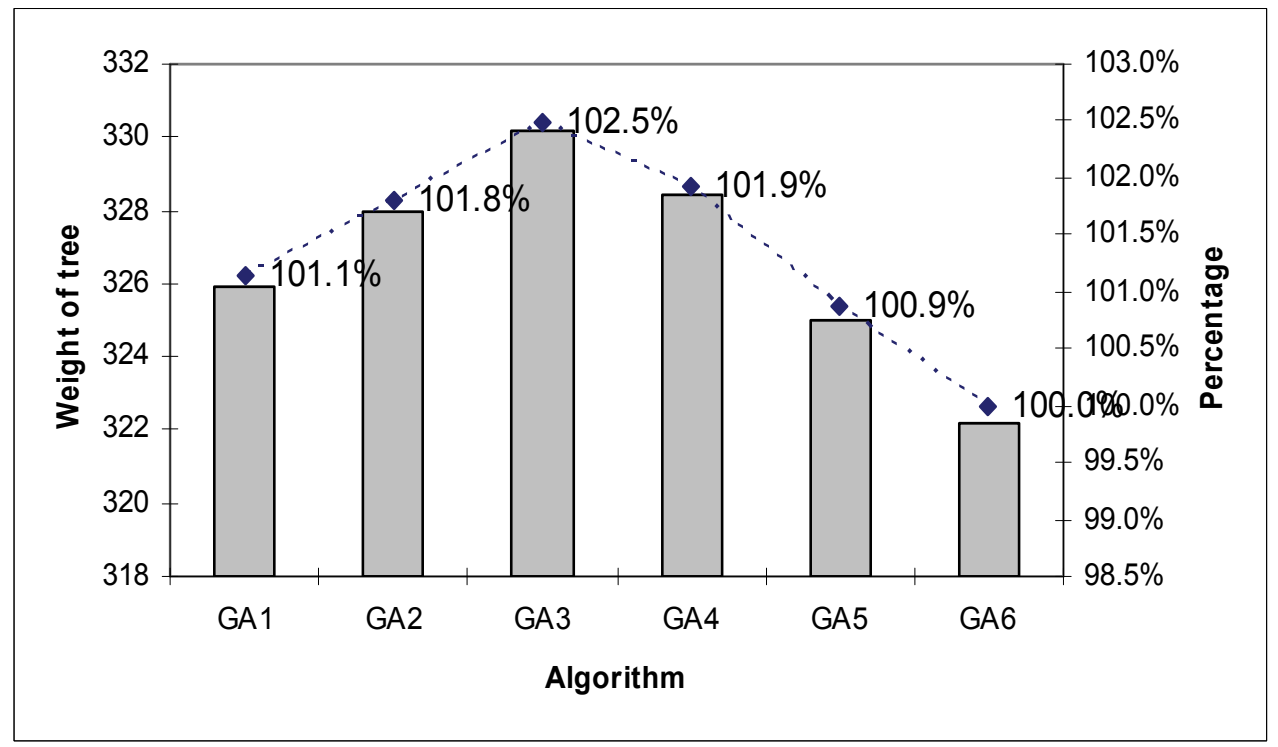

Fig. 18. Comparision between the best solution found by found by $G A_{1}, G A_{2}, G A_{3}, G A_{4}, G A_{5}$, $\mathrm{GA}_{6}$ on all the problem instance (20 instances) 


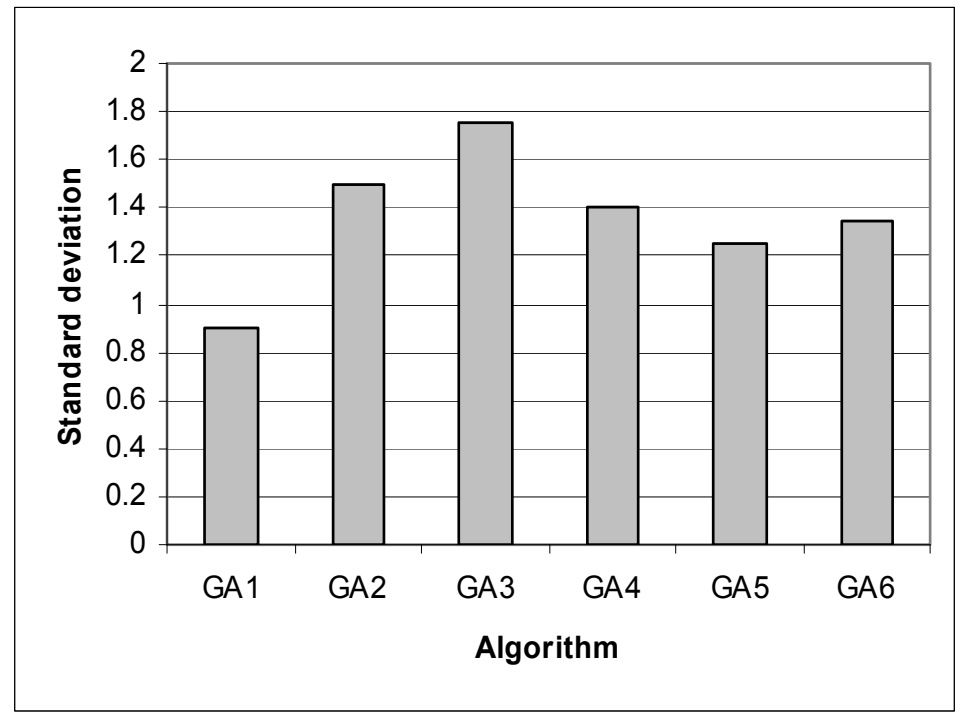

Fig. 19. Comparision between the standard deviation of the solution found by $G A_{1}, G A_{2}$, $G A_{3}, G A_{4}, G A_{5}, G_{6}$ on all the problem instance (20 instances)

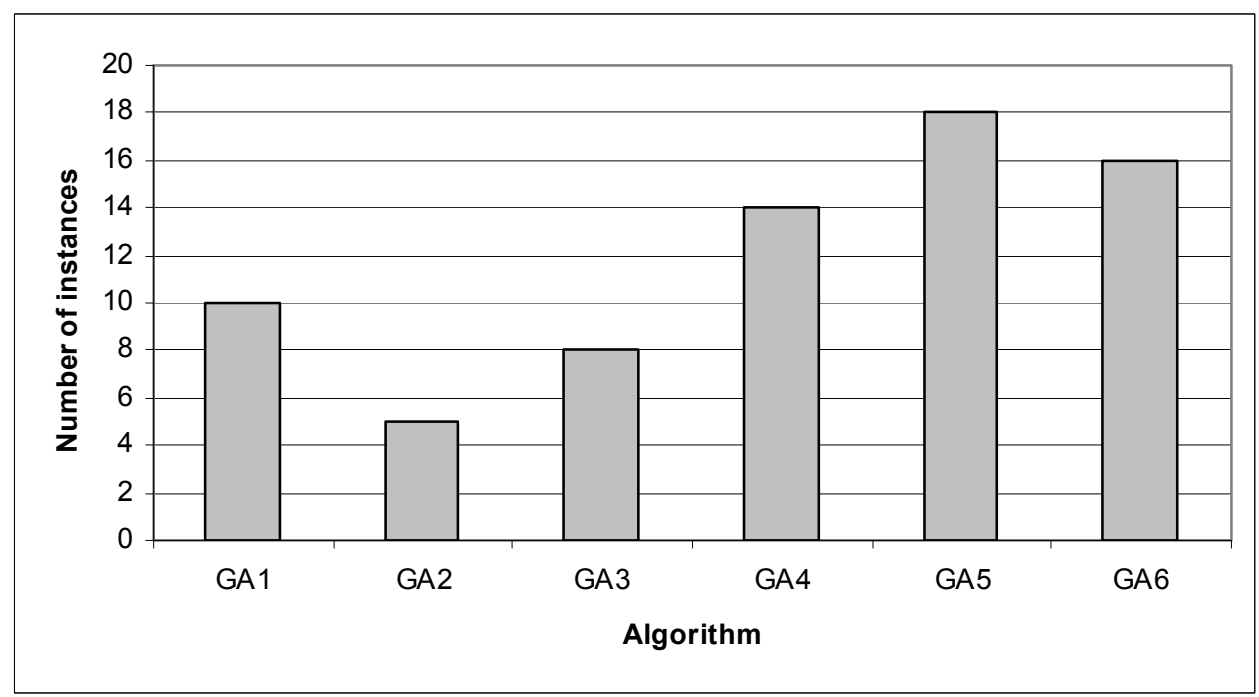

Fig. 20. Number of instances found best result by $G A_{1}, G A_{2}, G A_{3}, G A_{4}, G A_{5}, G A_{6}$ on all the problem instance (20 instances) 


\section{Conclusion}

We have introduced the heuristic algorithm for solving BDMST problem, called CBRC. The experiment shows that $C B R C$ have best result than the other known heuristic algorithm for solving BDMST prolem on Euclidean instances. The best solution found by the genetic algorithm which uses best heuristic algorithm or only one heuristic algorithm for initialization the population is not better than the best solution found by the genetic algorithm which uses mixed heuristic algorithms (randomized heuristic algorithm and greddy randomized heuristic algorithm). The solution found by the genetic algorithm which uses mixed heuristic algorithm for initialization always is the best result.

\section{References}

M.R. Garey and D.S.Johnson (1979), Computers and Intractability: A Guide to the Theory of NP-Completeness.

K. Raymond (1989), “A Tree-based Algorithm for Distributed Mutual Exclusion”, ACM Transactions on Computer Systems, 7 (1), 1989, pp. 61-77.

K. Bala, K. Petropoulos (1993), and T. E. Stern, "Multicasting in a linear Lightwave Network", in Proceedings of IEEE INFOCOM'93, 1993, pp. 1350-1358

C.C. Palmer and A. Kershenbaum (1994), "Representing Trees in Genetic Algorithms", in Proceedings of The First IEEE Conference on Evolutionary Computation, pp. 379384

N.R.Achuthan, L.Caccetta, P.Caccetta, and A. Geelen (1994), “Computational Methods for the Diameter Restricted Minimum Weight Spanning Tree Problem", Australian Journal of Combinatorics, 10, pp.51-71.

NA. Bookstein and S. T. Klein (1996), " Compression of Correlated Bit-Vectors", Information Systems, 16 (4), pp. 387-400.

G. Kortsarz and D. Peleg (1997), "Approximating Shallow-light Trees”, in Proceedings of the 8th Symposium on Discrete Algorithms, pp. 103-110.

A. Abdalla, N. Deo, and P. Gupta (2000), "Random-tree Diameter and the Diameter Constrained MST", in Proceedings of Congress on Numerantium, pp. 161-182.

J.Gottlieb, B.A.Julstrom, F.Rothlauf, and G.R.Raidl (2001), “Prufer Numbers: A Poor Representation of Spanning Trees for Evolutionary Search", in Proceedings of the Genetic and Evolutionary Computation Conference (GECCO'2001).

A. Abdalla (2001), "Computing a Diameter-constrained Minimum Spanning Tree", PhD Dissertation, The School of Electrical Engineering and Computer Science, University of Central Florida.

G.R. Raidl and B.A. Julstrom (2003), “Edge-sets: An Effective Evolutionary Coding of Spanning Trees", IEEE Transactions on Evolutionary Computation, 7, pp.225-239.

G.R. Raidl and B.A. Julstrom, (2003) "Greedy Heuristics and an Evolutionary Algorithm for the Bounded-Diameter Minimum Spanning Tree Problem", in Proceeding of the ACM Symposium on Applied Computing, pp. 747-752.

B.A. Julstrom, G.R. Raidl (2003), “A Permutation Coded Evolutionary for the Bounded Diameter Minimum Spanning Tree Problem, in Proceedings of the Genetic and Evolutionary Computation Conference (GECCO'2003), pp.2-7. 
B.A. Julstrom (2004), “Encoding Bounded Diameter Minimum Spanning Trees with Permutations and Random Keys", in Proceedings of Genetic and Evolutionary Computational Conference (GECCO'2004).

L Gouveia, T.L. Magnanti and C. Requejo (2004), “A 2-Path Approach for Odd Diameter Constrained Minimum Spanning and Steiner Trees", Network, 44 (4), pp. 254-265.

M. Gruber and G.R. Raidl (2005), "A New 0-1 ILP Approach for the Bounded Diameter Minimum Spanning Tree Problem, in Proceedings of the 2nd International Network Optimization Conference.

M. Gruber and G.R. Raidl (2005), "Variable Neighbourhood Search for the Bounded Diameter Minimum Spanning Tree Problem, in Proceedings of the 18th Mini Euro Conference on Variable Neighborhood Search, Spain.

M. Gruber, J. Hemert, and G.R. Raidl (2006), "Neighbourhood Searches for the Bounded Diameter Minimum Spanning Tree Problem Embedded in a VNS, EA and ACO", in Proceedings of Genetic and Evolutionary Computational Conference (GECCO'2006).

F. Rothlauf (2006), Representations for Genetic and Evolutionary Algorithms, 2nd Edition, Springer-Verlag.

Nguyen Duc Nghia and Huynh Thi Thanh Binh (2007), “A New Recombination Operator for Solving Bouded Diameter Minimum Spanning Tree Problem", in Proceedings of RIVF'2007, LNCS.

A. Singh and A.K. Gupta (2007), “An Impoved Heuristic for the Bounded Diameter Minimum Spanning Tree Problem, Journal of Soft Computing, 11, pp. 911-921.

G. Kortsarz and D. Peleg (1999), "Approximating the Weight of Shallow Steiner Trees", Discrete Application Mathematics, 93, 1999, pp. 265-285.

R. Prim (1957), "Shortest Connection Networks and Some Generalization", Bell System Technical Journal, 36, pp. 1389-1401.

Huynh Thi Thanh Binh, Nguyen Xuan Hoai, R.I Ian McKay (2008a), “A New Hybrid Genetic Algorithm for Solving the Bounded Diameter Minimum Spanning Tree Problem", Proceedings of IEEE World Congress on Computational Intelligence, Hong Kong, LNCS

Huynh Thi Thanh Binh, Nguyen Xuan Hoai, R.I Ian McKay, Nguyen Duc Nghia (2008b), "A new heuristic for the bouded diameter minimum spanning tree problem : some preliminary results", Conference on Artificial Intelligence PRICAI 2008, submitted 


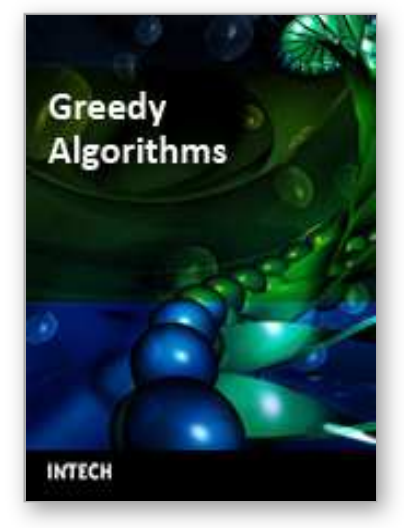

\author{
Greedy Algorithms \\ Edited by Witold Bednorz
}

ISBN 978-953-7619-27-5

Hard cover, 586 pages

Publisher InTech

Published online 01, November, 2008

Published in print edition November, 2008

Each chapter comprises a separate study on some optimization problem giving both an introductory look into the theory the problem comes from and some new developments invented by author(s). Usually some elementary knowledge is assumed, yet all the required facts are quoted mostly in examples, remarks or theorems.

\title{
How to reference
}

In order to correctly reference this scholarly work, feel free to copy and paste the following:

Nguyen Duc Nghia and Huynh Thi Thanh Binh (2008). Heuristic Algorithms for Solving Bounded Diameter Minimum Spanning Tree Problem and Its Application to Genetic Algorithm Development, Greedy Algorithms, Witold Bednorz (Ed.), ISBN: 978-953-7619-27-5, InTech, Available from:

http://www.intechopen.com/books/greedy_algorithms/heuristic_algorithms_for_solving_bounded_diameter_mi nimum_spanning_tree_problem_and_its_application_

\section{INTECH}

open science | open minds

\author{
InTech Europe \\ University Campus STeP Ri \\ Slavka Krautzeka 83/A \\ 51000 Rijeka, Croatia \\ Phone: +385 (51) 770447 \\ Fax: +385 (51) 686166 \\ www.intechopen.com
}

\author{
InTech China \\ Unit 405, Office Block, Hotel Equatorial Shanghai \\ No.65, Yan An Road (West), Shanghai, 200040, China \\ 中国上海市延安西路65号上海国际贵都大饭店办公楼 405 单元 \\ Phone: +86-21-62489820 \\ Fax: $+86-21-62489821$
}


(C) 2008 The Author(s). Licensee IntechOpen. This chapter is distributed under the terms of the Creative Commons Attribution-NonCommercialShareAlike-3.0 License, which permits use, distribution and reproduction for non-commercial purposes, provided the original is properly cited and derivative works building on this content are distributed under the same license. 